\title{
NEUROTOXICIDADE DO OXAMNIQUINE NO TRATAMENTO DA INFECÇÃO HUMANA PELO SCHISTOSOMA MANSONI
}

\author{
Silvino Alves de CARValHo (1), Maria Aparecida SHIKANAI-YASUDA (2), Vicente AMATO NETO (3), \\ Mario SHIRoMa (4) e Francisco José Carchedi LUCCAS (5)
}

\begin{abstract}
R E S U M O
Foram tratados com oxamniquine (dose oral única de 12,5 a $15 \mathrm{mg}$ e 15 a $20 \mathrm{mg} / \mathrm{kg}$ de peso, para maiores e menores de 15 anos respectivamente) 180 indivíduos com esquistossomose mansoni, matriculados na Clínica de Doenças Infecciosas e Parasitárias do Hospital das Clínicas da Faculdade de Medicina da Universidade de São Paulo. As idades variaram de 5 a 65 anos e as formas clinicoevolutivas prevalentes foram a intestinal e hepatointestinal. Os principais efeitos colaterais neuropsiquiátricos foram: sonolência $(50,6 \%)$, tontura $(41,1 \%)$, cefaléia $(16,1 \%)$, amnésia transitória $(2,2 \%)$, alterações de comportamento $(1,7 \%)$, tremores $(1,1 \%)$ e convulsão $(1,1 \%)$. Em 20 indivíduos foi avaliada a neurotoxicidade da droga através de eletroencefalografia, antes e após o tratamento. $\operatorname{Em~} 3$ (15\%), foram detectados alteraçōes no traçado, sem contudo apresentarem manifestaçóes clínicas neuropsiquiátricas. Os resultados demonstram ser o oxamniquine determinante de efeitos tóxico-colaterais na esfera neuropsiquiátrica.
\end{abstract}

\section{N T R O D U G A O}

RICHARDS \& FOSTER ${ }^{7}$ descreveram em 1969 uma série de derivados aminometiltetrahidroquinoleínicos com ação esquistossomicida. Desses compostos o que apresentou maior atividade foi o UK-3883 (2-isopropilaminometil-6metil-7-nitro-1,2,3,4,-tetrahidroquinoleína). Este composto, quando metabolizado organicamente sofre uma hidroxilação no grupamento 6-metil, originando o 6-hidroxometil-2-isopropilaminometil-7-nitro, 1,2,3,4,-tetrahidroquinoleína. Foi denominado inicialmente de UK-4271 e posteriormente, com o nome genérico de oxamniquine.

Este composto apresenta elevada ação esquistossomicida e sua obtenção em escala in- dustrial se processa através da hidroxilação do UK-3883, pela atividade biológica do Aspergillus selerotiorum.

KAYE \& WOOLHOUSE 11 , estudando o metabolismo do oxamniquine em animais de laboratório e voluntários humanos, concluíram que o medicamento é bem absorvido por via oral e parenteral e que a via de excreção é a urinária. através de dois metabólitos (6-carboxi e 2-ácj do carboxílico). A meia vida, medida atravis de liberação radioativa no sangue após adıninistração oral é de 2 a 6 horas.

$\mathrm{O}$ oxamniquine foi utilizado inicialmente por via parenteral na dose única de $7,5 \mathrm{mg} / \mathrm{kg}$.

(1) Professor-assistente da Clínica de Doenças Infecciosas e Parasitárias da Faculdade de Medicina da Universidade de São Paulo

(2) Professora-assistente doutora da Clínica de Doenças Infecciosas e Parasitárias da Faculdade de Medicina da Universidade de São Paulo

(3) Professor-titular da Clínica de Doenças Infecciosas e Parasitárias da Faculdade de Medicina da Universidade de São Paulo

(4) Professor-adjunto da Clínica de Doenças Infecciosas e Parasitárias da Faculdade de Medicina da Universidade de São Paulo

(5) Médico-assistente do Serviço de Eletroencefalografia da Clínica Neurológica do Hospital das Clínicas da Faculdade de Medicina da Universidade de São Paulo 
CARVALHO, S. A. de; SHIKANAI-YASUDA, M. A.; AMATO NETO, V.; SHIROMA, M. \& LUCCAS, F. J. C, - Neurotoxicidade do oxamniquine no tratamento da infeç̧ão humana pelo s. mansoni. Rev. Inst. Med. trop. Sáo Paulo 27. $132-142,1985$

Por causar forte dor e necrose no local da aplicação, passou a ser administrado por via oral $(12,5$ a $15 \mathrm{mg} / \mathrm{kg}$ para adultos e $20 \mathrm{mg} / \mathrm{kg}$ para crianças).

De modo geral, os trabalhos existentes em literatura, quase que invariavelmente, caracterizam a droga como altamente eficaz e desprovida de toxicidade, esta última objeto de dis. cussão do presente trabalho.

\section{MATERIAL E METTODOS}

O material em estudo constituiu-se de 180 pacientes com esquistossomose mansoni, de am bos os sexos, com idade variável de 5 a 65 anos, matriculados na Clínica de Doenças Infecciosas e Parasitárias do Hospital das Clínicas da Fa culdade de Medicina da Universidade de São Paulo. O diagnóstico da esquistossomose, na totalidade dos casos, foi realizado pela coproscopia qualitativa e quantitativa. A grande maioria dos pacientes era assintomática (Tabela I) e a associação mórbida prevalente foi a hipertensão arterial, seguida de outras com menor freqüência (Tabela II).

As formas clínico-evolutivas predominantes foram a intestinal (I) e hepatointestinal (HI). As formas hepatoesplênica compensada (HEC) e descompensada (HED), ocorreram com un número muito menor de individuos (Tabela III). A coproscopia quantitativa mostrou serem leves a maioria das infecçōes pelo $\mathbf{S}$. mansoni (Tabela IV).

O oxamniquine foi administrado na dose de 12,5 a $15 \mathrm{mg} / \mathrm{kg}$ para maiores de 15 anos e 16 a $20 \mathrm{mg} / \mathrm{kg}$ de peso para menores de 15 anos. Após a administração do medicamento, os pa. cientes eram colocados em repouso relativo por 4 horas, sempre sob supervisão da equipe de enfermagem, orientada no sentido de anotar qualquer efeito colateral que, eventualmente, pudesse surgir no período de observação. Quando do primeiro retorno ( 1 semana), os pacientes eram submetidos a rigorosa anamnese, no sentido de se detectar possíveis efeitos colate. rais de aparecimento mais tardio.

Para se detectar possiveis agravos ao sistema nervoso central, foram realizados em 20 pacientes (com forma clínico-evolutiva intestinal e hepatointestinal) estudo eletroencefalográfico, antes e mais ou menos 2 horas após o tratamento.
T A B E I A I

Distribuição do no e \% dos pacientes segundo os sintomás referidos antes do tratamento com oxamniquine

\begin{tabular}{|c|c|c|}
\hline Sintomas (*) & N.o & $(\%)$ \\
\hline Sem queixas & 103 & 57,2 \\
\hline Cólicas abdominais & 34 & 18,9 \\
\hline Obstipação & 15 & 8,3 \\
\hline Diarréia & 14 & 7,8 \\
\hline Anorexia & 13 & 7,2 \\
\hline Fraqueza & 13 & 7,2 \\
\hline Disenteria & 7 & 3,9 \\
\hline Tonturas & 6 & 3,3 \\
\hline Empachamento & 6 & 3,3 \\
\hline Cefaléia & 6 & 3,3 \\
\hline Aumento do abdome & 5 & 2,8 \\
\hline Hematemese & 4 & 2,2 \\
\hline Náuseas & 3 & 1,7 \\
\hline Disfagia & 3 & 1,7 \\
\hline Hipodesenvolvimento & 2 & 1,1 \\
\hline Dispnéia & 2 & 1,1 \\
\hline Melena & 2 & 1,1 \\
\hline Epistaxis & $I$ & 0,6 \\
\hline Sonambulismo & 1 & 0,6 \\
\hline Enurese noturna & 1 & 0,6 \\
\hline
\end{tabular}

(*) Alguns pacientes apresentaram um ou mais sintomas

T A B E L A II

Distribuição do n. ${ }^{\circ}$ e dos pacientes tratados com oxamniquine segundo doenças intercorrentes

\begin{tabular}{|c|c|c|}
\hline Doenças intercorrentes $(*)$ & N.o & $(\%)$ \\
\hline Hipertensão arterial & 21 & 11,7 \\
\hline Cardiopatia chagásica & 6 & 3,3 \\
\hline Outras cardiopatias & 6 & 3,3 \\
\hline Disritmia cerebral & 6 & 3,3 \\
\hline Megaesôfago & 4 & 2,2 \\
\hline Bócio tóxico & 3 & 1,7 \\
\hline Bócio atóxico & 2 & 1,1 \\
\hline Anemia falciforme & 2 & 1,1 \\
\hline Obesidade & 2 & 1,1 \\
\hline Enurese noturna & 2 & 1,1 \\
\hline Hipodesenvolvimento & 2 & 1,1 \\
\hline Doença reumatoide & 1 & 0,6 \\
\hline Lupus eritematoso discóide & 1 & 0,6 \\
\hline Púrpura idiopática & 1 & 0,6 \\
\hline Neuropatia heredo-degenerativa & 1 & 0,6 \\
\hline Hérnia de hiato & 1 & 0,6 \\
\hline Trombose de art. retiniana & 1 & 0,6 \\
\hline Oligofrenia & 1 & 0,6 \\
\hline Doença de Hodgkin & 1 & 0,6 \\
\hline Insuficiência vértebro-basilar & 1 & 0,6 \\
\hline Prurido auto-tóxico & 1 & 0,6 \\
\hline Fístula do tireoglosso & 1 & 0,6 \\
\hline Psoríase & 1 & 0,6 \\
\hline Síndrome do túnel do carpo & 1 & 0,6 \\
\hline Osteoartrose & 1 & 0,6 \\
\hline Hematúria inespecífica & 1 & 0,6 \\
\hline Sem patologia intercorrente & 109 & 60,5 \\
\hline Total & 180 & 100 \\
\hline
\end{tabular}

(*) Patologias intercorrentes diagnosticadas que motivaram ou não a consulta 
CARVAlho, S. A, de; SHIKANAI-YASUDA, M. A.; AMATO NETO, V.; SHIROMA, M. \& LUCCAS, F. J. C. - Neurotoxicidađe do oxamniquine no tratamento da infeç̧ão humana pelo $S$. mansoni. Rev. Inst. Med. trop. São Paulo 27: 132-142, 1985.

T A B E L A III

Distribuição do $n .^{\circ}$ e $\%$ de pacientes tratados com oxamniquine segundo a faixa etária e formas clínicas

\begin{tabular}{|c|c|c|c|c|c|c|c|c|c|c|}
\hline \multirow{2}{*}{$\begin{array}{l}\text { Faixa } \\
\text { etária }\end{array}$} & \multicolumn{2}{|c|}{$I$} & \multicolumn{2}{|c|}{ HI } & \multicolumn{2}{|c|}{$\mathrm{HEC}$} & \multicolumn{2}{|c|}{ HED } & \multicolumn{2}{|c|}{ Total } \\
\hline & N.o & $(\%)$ & N.o & $(\%)$ & N.o & $(\%)$ & N.o & $(\%)$ & N.o & $(\%)$ \\
\hline $5-15$ & 11 & $(14,7)$ & 14 & $(19,4)$ & 12 & $(52,2)$ & - & - & 37 & $(20,6)$ \\
\hline $16-25$ & 29 & $(38,7)$ & 26 & $(36,1)$ & 6 & $(26,1)$ & 4 & $(40,0)$ & 65 & $(36,1)$ \\
\hline $26-35$ & 19 & $(25,3)$ & 17 & $(23,7)$ & 3 & $(13,0)$ & 4 & $(40,0)$ & 43 & $(23,9)$ \\
\hline $36-45$ & 9 & $(12,0)$ & 6 & $(8,3)$ & 2 & $(8,7)$ & 2 & $(20,0)$ & 19 & $(10,5)$ \\
\hline $46-55$ & 5 & $(6,7)$ & 4 & $(5,5)$ & - & - & - & - & 9 & $(5,0)$ \\
\hline 56 e mais & 2 & $(2,6)$ & 5 & $(6,7)$ & - & - & - & - & 7 & $(3,9)$ \\
\hline Total & 75 & $(100,0)$ & 72 & $(100,0)$ & 23 & $(100,0)$ & 10 & $(100,0)$ & 180 & $(100,0)$ \\
\hline
\end{tabular}

T A B E I A IV

Distribuição do $n .^{\circ}$ e $\%$ de pacientes tratados com oxamniquine segundo as formas clínicas e contagem quantitativa dos ovos de S. mansoni (n.० de ovos/g de fezes) $\left(^{*}\right)$

\begin{tabular}{|c|c|c|c|c|c|c|c|c|c|c|}
\hline \multirow{2}{*}{$\begin{array}{l}\text { N.o de } \\
\text { ovos } \\
\text { g de } \\
\text { fezes }\end{array}$} & \multicolumn{2}{|c|}{ I } & \multicolumn{2}{|c|}{ HI } & \multicolumn{2}{|c|}{$\mathrm{HEC}$} & \multicolumn{2}{|c|}{ HED } & \multicolumn{2}{|c|}{ Total } \\
\hline & N.o & $(\%)$ & N. ${ }^{\circ}$ & $(\%)$ & N.o & $(\%)$ & N.o & $(\%)$ & N.o & $(\%)$ \\
\hline $0-100$ & 23 & $(47,0)$ & 27 & $(54,0)$ & 6 & $(43,0)$ & 4 & $(57,0)$ & 60 & $(50,0)$ \\
\hline $101-200$ & 13 & $(26,5)$ & 8 & $(16,0)$ & 5 & $(35,7)$ & 1 & $(14,3)$ & 27 & $(22,5)$ \\
\hline $201-300$ & 3 & $(6,1)$ & 4 & $(8,0)$ & 1 & $(7,1)$ & - & - & 8 & $(6,7)$ \\
\hline $301-400$ & 1 & $(2,0)$ & 6 & $(12,0)$ & - & - & - & - & 7 & $(5,8)$ \\
\hline $401-500$ & 4 & $(8,2)$ & 1 & $(2,0)$ & - & - & 1 & $(14,3)$ & 6 & $(5,0)$ \\
\hline 501 e mais & 5 & $(10,2)$ & 4 & $(8,0)$ & 2 & $(14,2)$ & 1 & $(14,3)$ & 12 & $(10,0)$ \\
\hline Total & 49 & $(100,0)$ & 50 & $(100,0)$ & 14 & $(100,0)$ & 7 & $(100,0)$ & 120 & $(100,0)$ \\
\hline
\end{tabular}

(*) 60 indivíduos não realizaram a coproscopia quantitativa

T A B E L A V

Distribuição do n. e \% dos pacientes que apresentaram, efeitos tóxico-colaterais neuropsiquiátricos após tratamento com oxamniquine

\begin{tabular}{lcr}
\hline $\begin{array}{l}\text { Efeitos colaterais } \\
\text { neuropsiquiátricos }\left(^{*}\right)\end{array}$ & N. ${ }^{\circ}$ & $(\%)$ \\
\hline Sonolência & 91 & 50,6 \\
Tonturas & 74 & 41,1 \\
Cefaléia & 29 & 16,1 \\
Amnésia transitória & 4 & 2,2 \\
Alteração de comportamento & 3 & 1,7 \\
Tremores & 2 & 1,1 \\
Convulsão & 2 & 1,1
\end{tabular}

$\left(^{*}\right)$ Alguns pacientes apresentaram um ou mais sintomas dos relacionados

\section{RESULTADOS}

O oxamniquine de modo geral, foi bem tolerado $e$ as reações colaterais surgiram mais freqüentemente entre 1 a 3 horas após a tomada do medicamento. Em nosso estudo somente 5 pacientes apresentaram manifestações im- portantes atribuíveis ao medicamento, com duração superior a 12 horas, e se caracterizaram, principalmente, por distúrbios neuropsiquiátricos e digestivos. Na maioria dos pacientes as manifestações foram leves ou de moderada intensidade, traduzidas por: sonolência $(50,1 \%)$, tonturas $(40,1 \%)$, cefaléia $(16,1 \%)$ e tremores $(1,1 \%)$, (Tabela V). Entretanto sete pacientes desenvolveram reações neuropsiquiátricas severas, que a seguir serão analisadas cada caso em particular.

CASO 1 - A.R.A., 17 anos, branca, feminina, portadora de forma intestinal e sem nenhuma patologia intercorrente. $\mathrm{Na}$ anamnese apresentava "crises de esquecimento" que, se valorizadas, poderiam sugerir manifestações disrítmicas tipo pequeno mal. Nunca apresentou desmaios, com ou sem perda de consciência, ou crise convulsiva. Foi medicada em jejum com $750 \mathrm{mg}$ de oxamniquine. Noventa minutos após a ingestão do medicamento, apresentou crise 
CARVALHO, S. A. de; SHIKANAI-YASUDA, M. A.; AMATO NETO, V.; SHIROMA, M. \& LUCCAS, F. J. C. - NeUrotoxici. dade do oxamniquine no tratamento da infeç̧ão humana pelo $S$. mansoni. Rev. Inst. Med. trop. São Paulo 2\%: $132-142,1985$

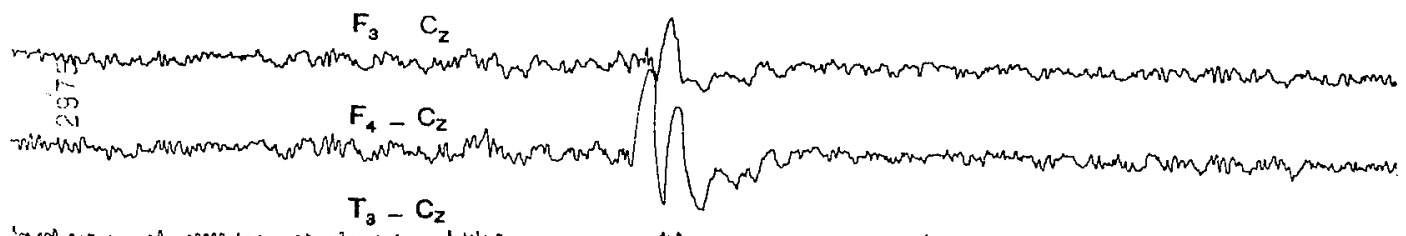

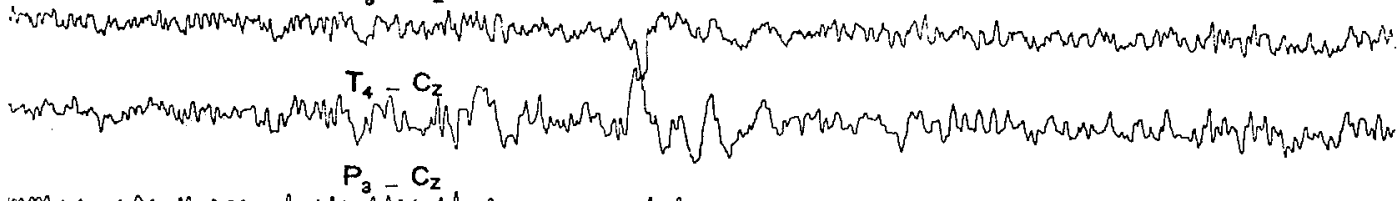

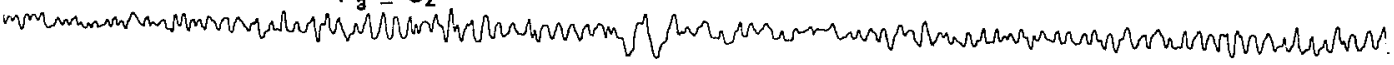

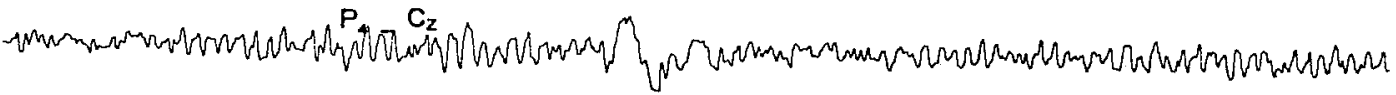

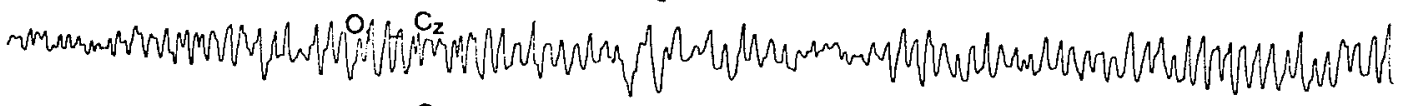

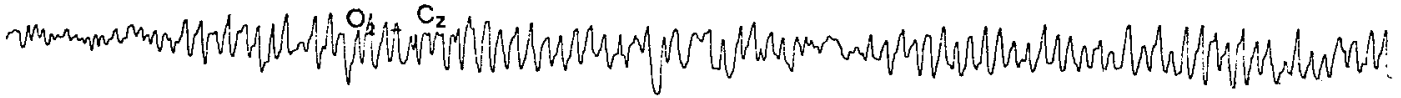

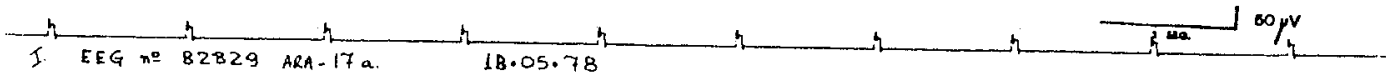

Fig. 1(a) - EEG realizado mais ou menos 90 minutos após a crise convulsiva: anormalidades paroxísticas de ca ráter irritativo anteriores (frontais) mais à đireita, por vezes com difusão. Ritmo de base normal para a idade

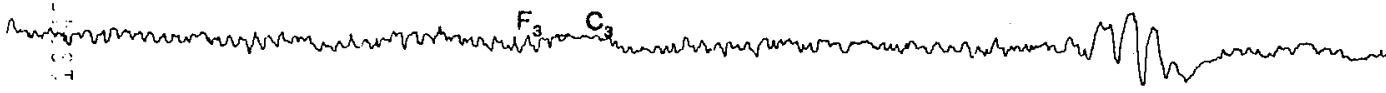

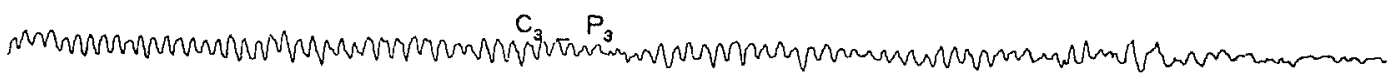

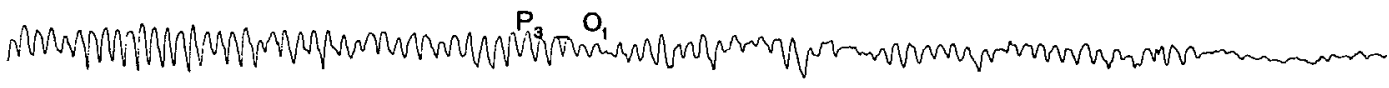

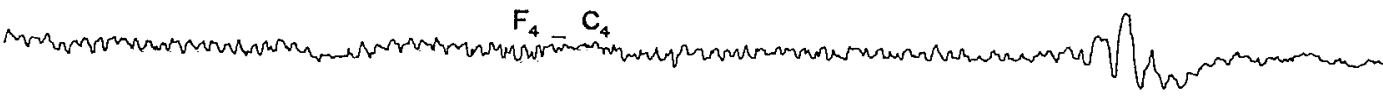

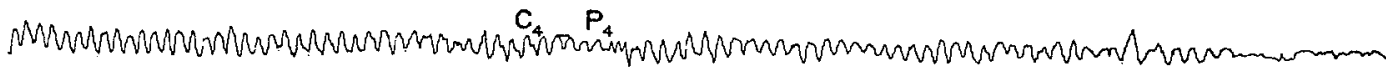

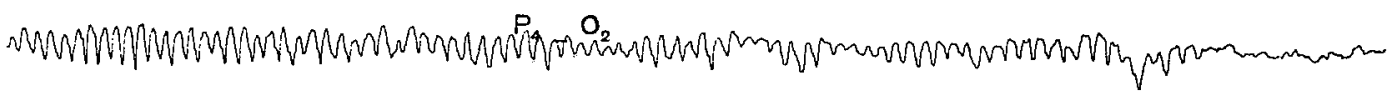

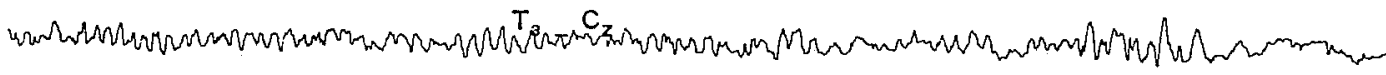

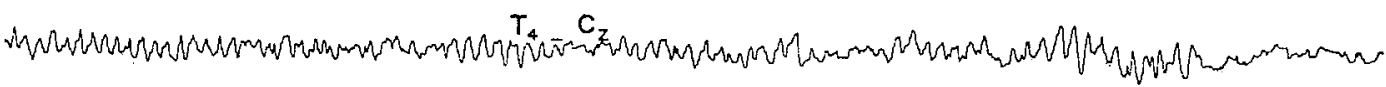

I. $t_{E \in G} x=83225$ ARA - 272 .

Fig. 1(b) - EEG realizados 14 e 28 dias após a crise convulsiva: o caráter anormal do EEG anterior praticamente inalterado

convulsiva, com abalos tônico-clônicos generalizados e perda da consciência, que duraram 15 minutos. No decorrer da crise convulsiva foi medicada com diazepínico. O eletroencefalograma realizado mais ou menos 90 minutos após a crise, revelou anormalidade paroxística de ca- 
CARVALHO, S. A. de; SHIRANAI-YASUdA, M. A.; AMATO NETO, V.; SHIROMA, M. \& LUCCAS, F. J. C. - Neurotoxicidade do oxamniquine no tratamento da infecção humana pelo $S$. mansoni. Rev. Inst. Med. trop. São Paulo 27: 132-142, 1985.

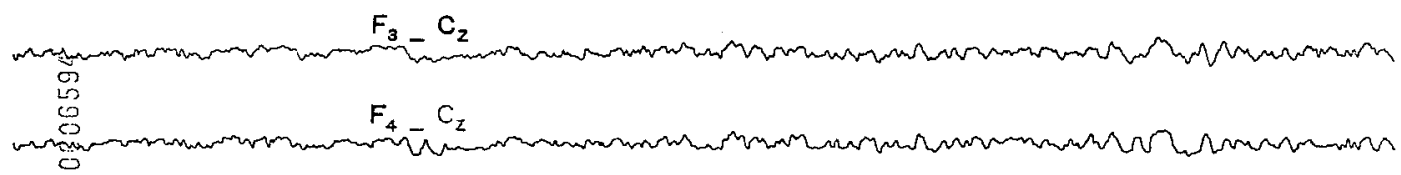

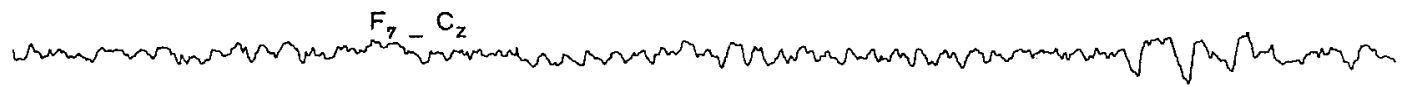

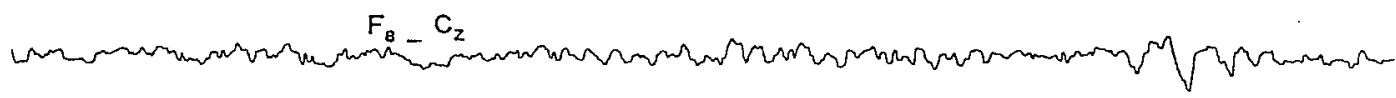

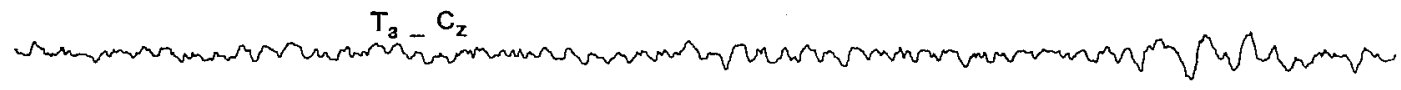

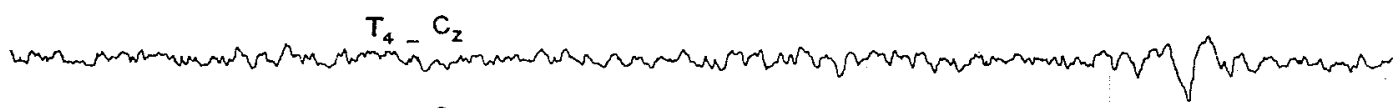

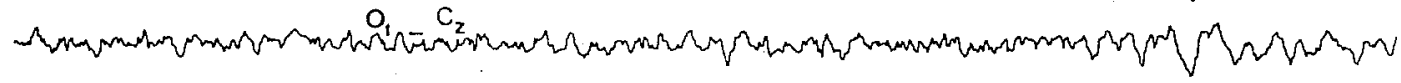

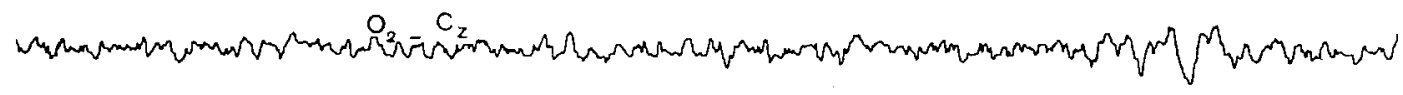

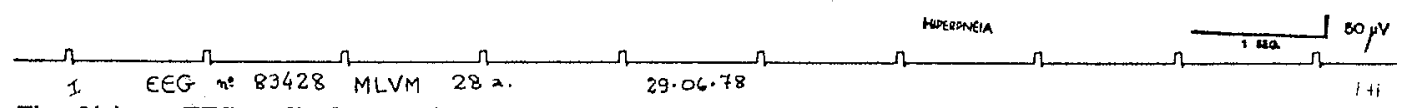

Fig. 2(a) - EEG realizado 90 minutos após a crise convulsiva: Sinais raros de anormalidades paroxísticas difusas frustras, melhor evidenciađas à ativação pela hịperpnéia
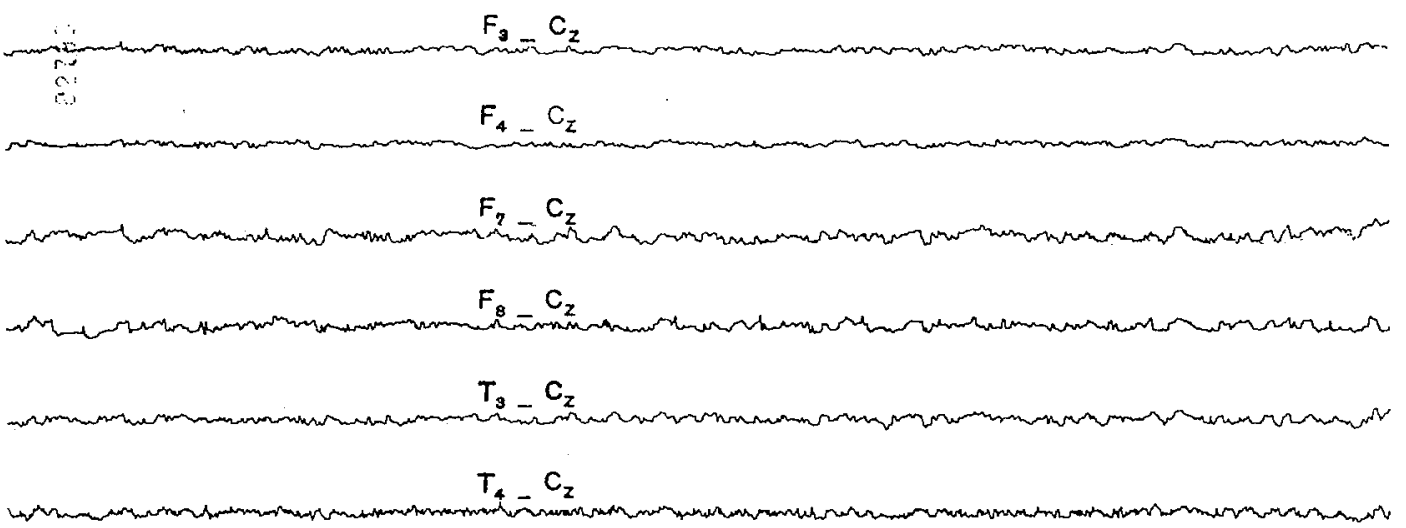

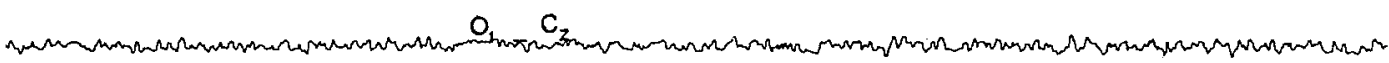

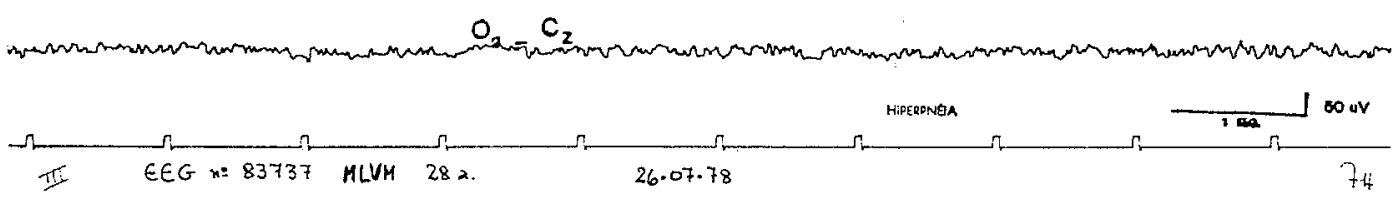

Fig. 2(b) - EEG realizado 7 e 28 dias após a crise convulsiva: EEG pequena amplitude, dentro dos limites de normalidade

ráter irritativo difuso (Fig. 1(a)). Dois eletroencefalogramas realizados 14 e 28 dias após a convulsão não apresentaram nenhuma alteracão no traçado em relação ao anterior (Fig. 1(b) ). Pelo padrão dos traçados e a sua per. sistência, sugeriu ser a paciente portadora de disritmia cerebral de base. 
CARVALHO, S. A. de; SHIKANAI-YASUDA, M. A.; AMATO NETO, V.; SHIROMA, M. \& LUCCAS, F. J. C. - Neurotoxicidađe do oxamniquine no tratamento da infecção humana pelo $S$. mansoni. Rev. Inst. Med. trop. São Paulo 27: 132-142, 1985 .

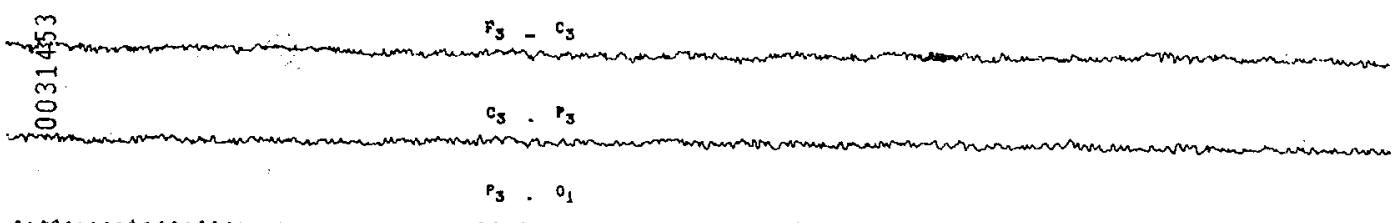

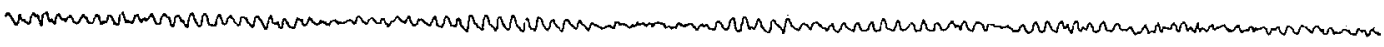

ríninin

$c_{4} \cdot P_{4}$

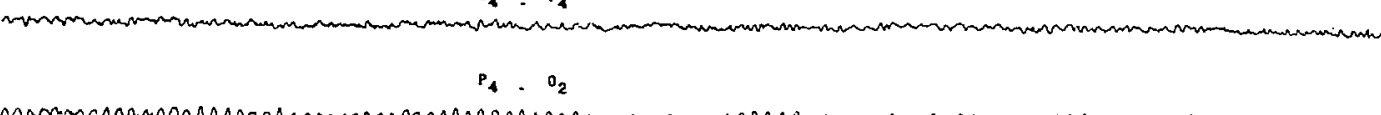

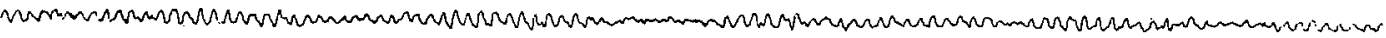

$\mathbf{T}_{\mathbf{3}} \ldots \mathbf{c}_{\mathbf{z}}$

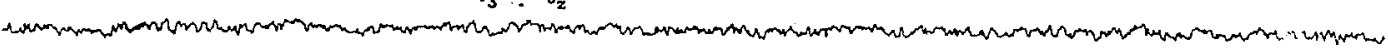

$T_{4} \cdot c_{2}$

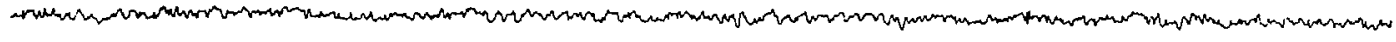

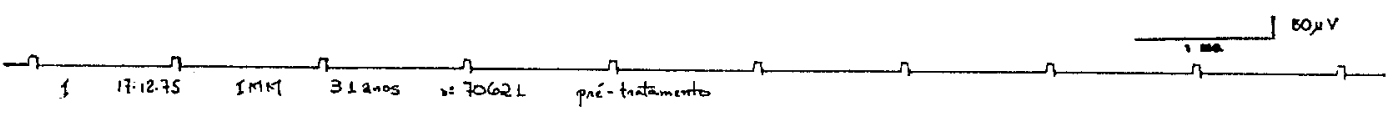

Fig. 3(a) - EEG sem anormalidades antes do tratamento

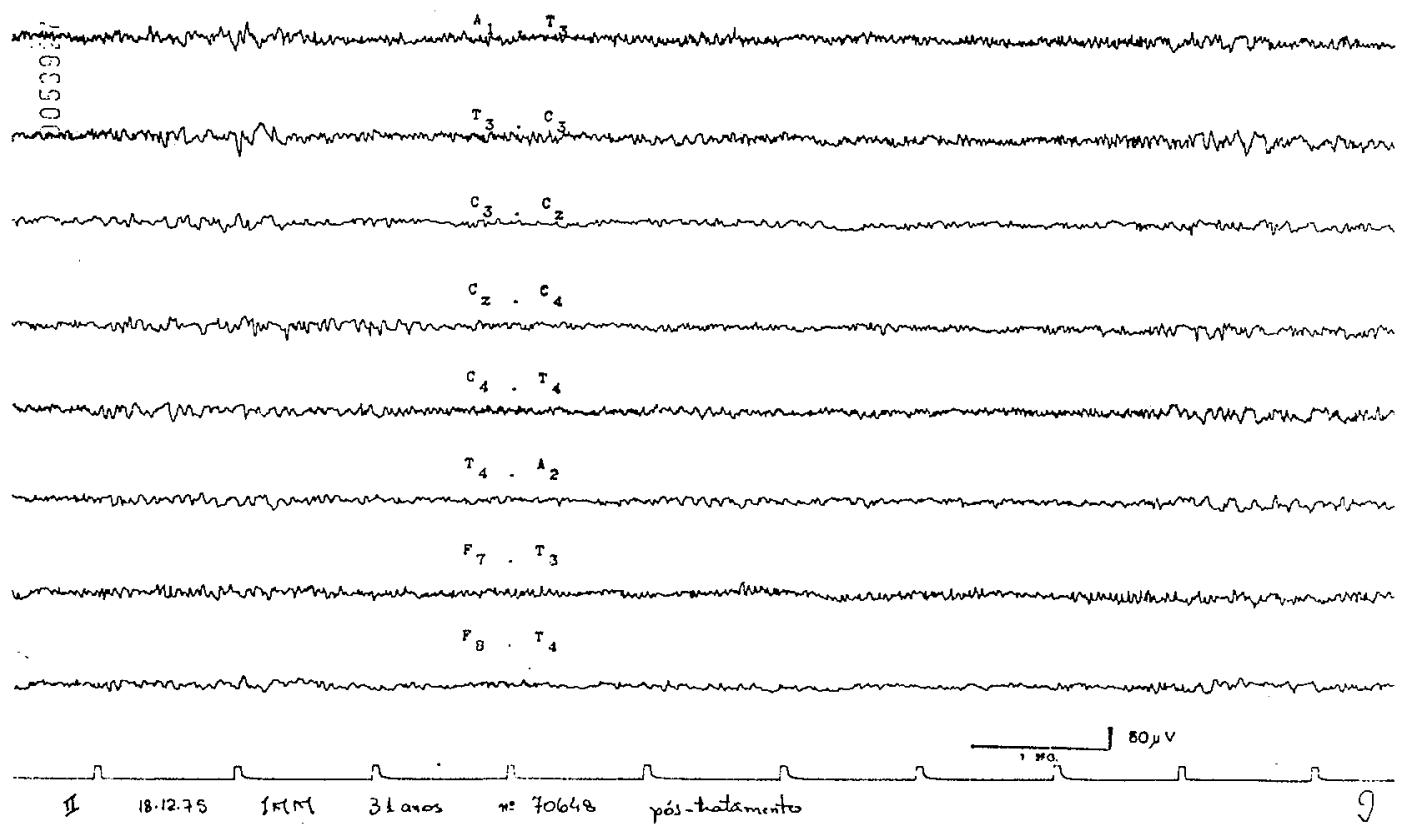

Fig. 3(b) - EEG apresentando atividade irritativa difusa, frustra, mais à esquerđa à hiperpnéia, mais ou menos 2 horas após a medicação

CASO 2 - M.L.V.M., 28 anos, branca, feminina, portadora de forma intestinal e sem nenhuma patologia intercorrente ou sintomas sugestivos de disritmia cerebral. A paciente re- cebeu $1000 \mathrm{mg}$ de oxamniquine, em jejum. Setenta minutos após, apresentou crise convulsiva com abalos tônico-clônicos generalizados, com perda da consciência que duraram $5 \mathrm{mi}$ - 
CARVAlHo, S. A. de; SHIKANAI-YASUdA, M. A.; AMATO NETO, V.; ShIROMA, M. \& LUCCAS, F. J. C. - Neurotoxici. dađe do oxamniquine no tratamento da infecçăo humana pelo S. mansoni. Rev." Inst. Med. trop. São Paiulo 27: 132-142, 1985.

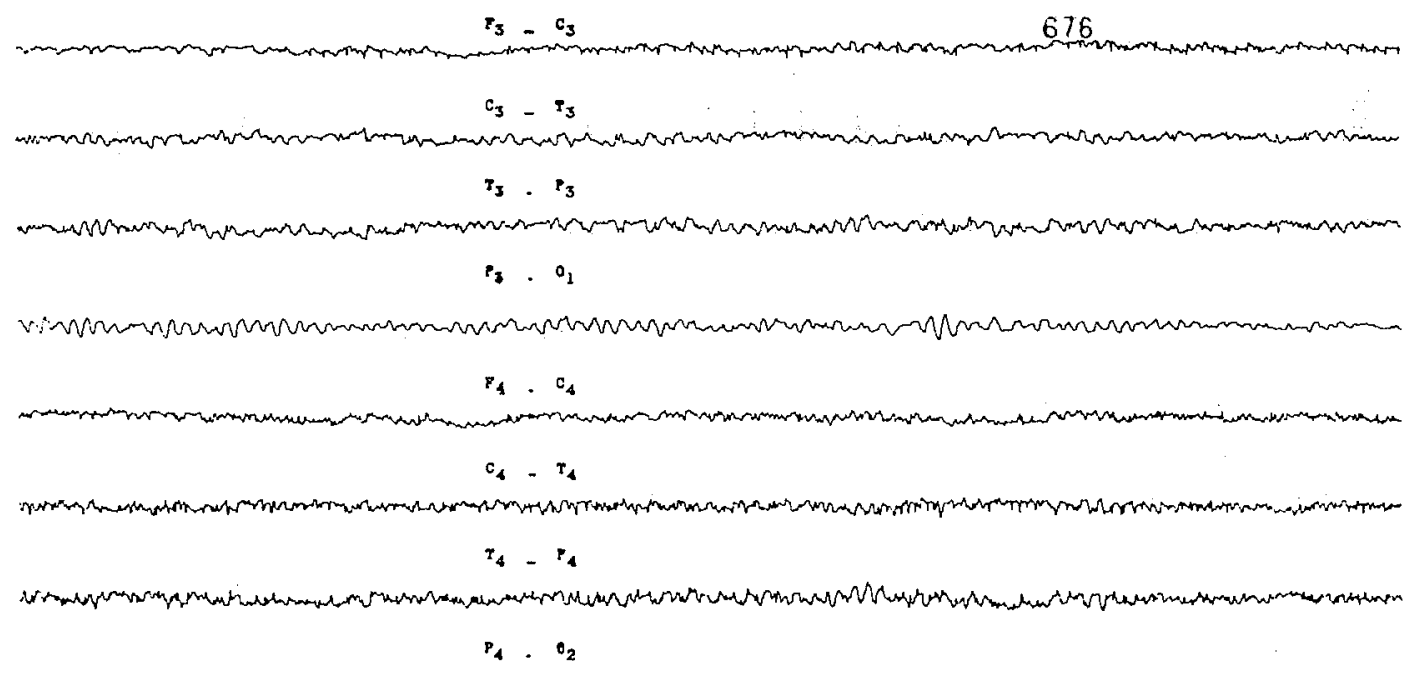

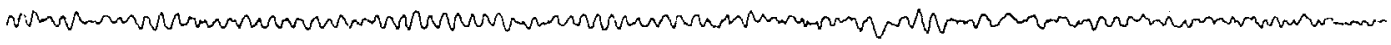

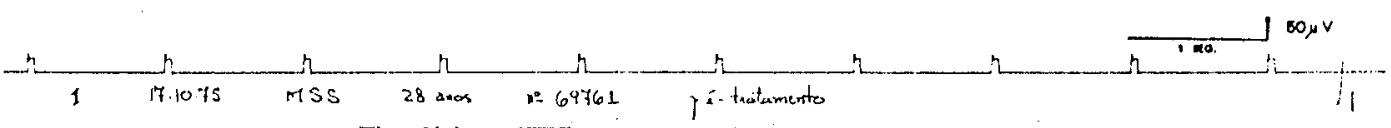

Fig. 4(a) - EEG sem anormalidades antes do tratamento

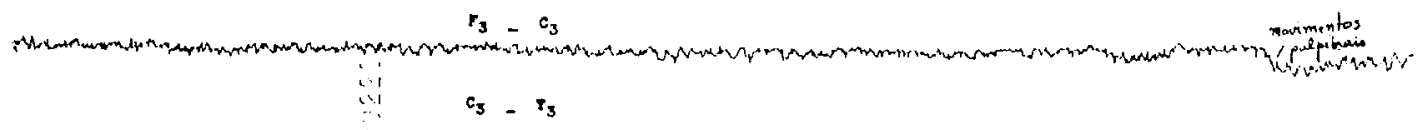

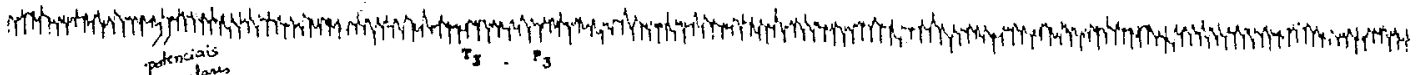

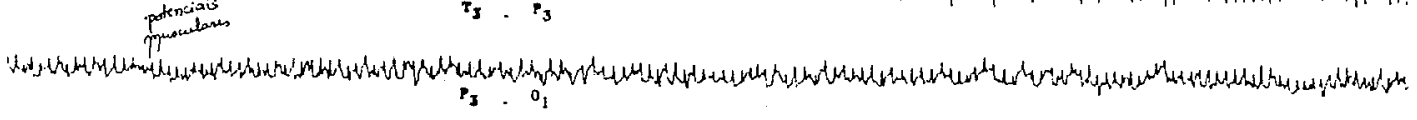

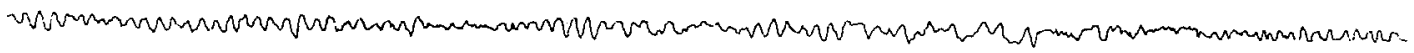

$r_{1} \cdot c_{4}$

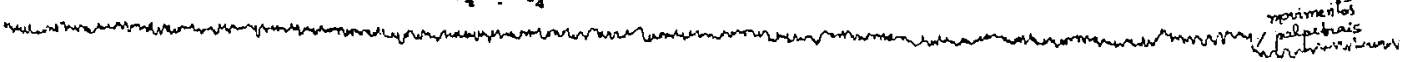

$c_{1}-T_{4}$

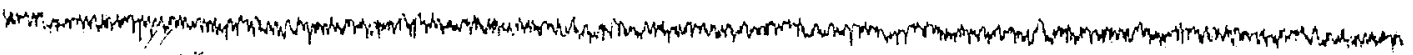
$\tau_{4}$ - Pot

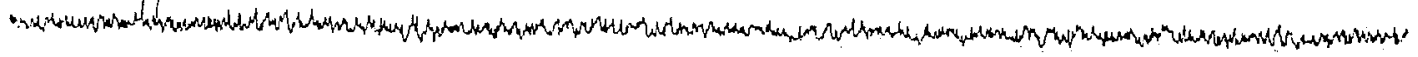

$P_{4} \cdot o_{2}$

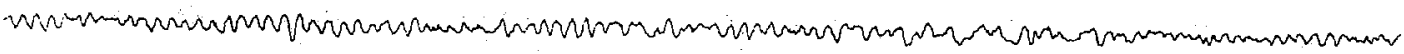

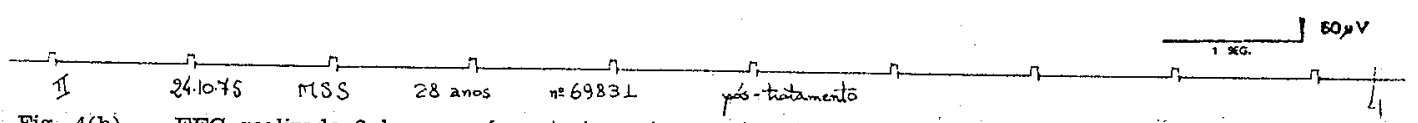

Fig. 4(b) - EEG realizado 2 horas após o tratamento, mostranđo sinais sugestivos de anormalidade paroxística frustra occipital

nutos. Durante a convulsão foi medicada com diazepínico. O eletroencefalograma realizado 90 minutos após a crise, revelou sinais de anormalidades paroxísticas difusas frustras, melhor evidenciando à hiperpnéia (Fig. 2(a)). Dois eletroencefalogramas realizados 7 e 28 dias após a crise convulsiva não apresentaram qualquèr alteração no traçado (Fig. 2(b)). 
CARVALHO, S. A. de; SHIKANAI-YASUDA, M. A.; AMATO NETO, V.; SHIROMA, M. \& LUCCAS, F. J. C. - Neurotoxicidade do oxamniquine no tratamento da infecção humana pelo S. mansoni. Rev. Inst. Med, trop. São Paulo 27: 132-142, 1985

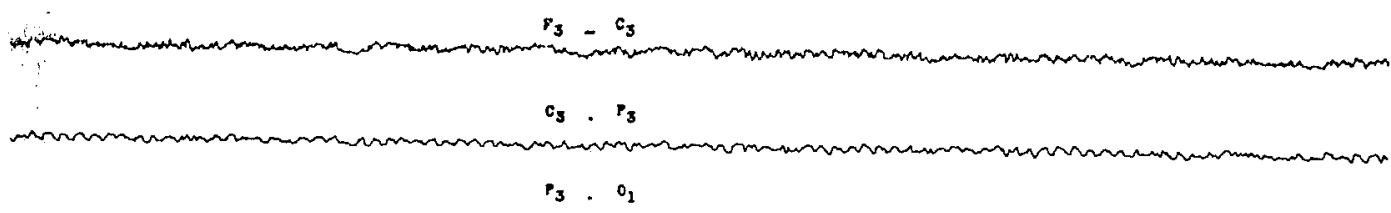

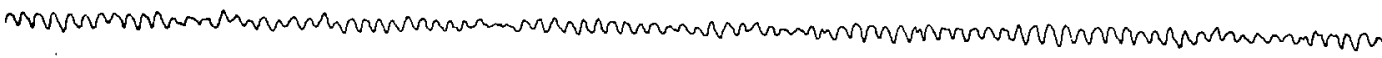

$r_{4} \cdot c_{4}$

$P_{4} \cdot B_{2}$

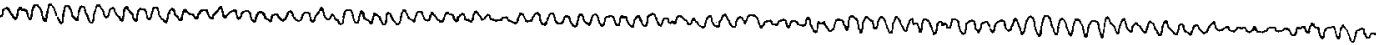

$T_{3} \cdot c_{2}$

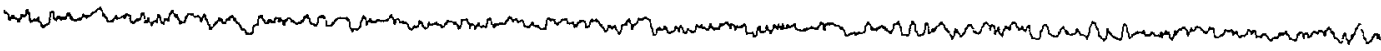

$T_{1} \cdot c_{z}$

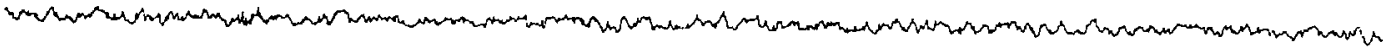

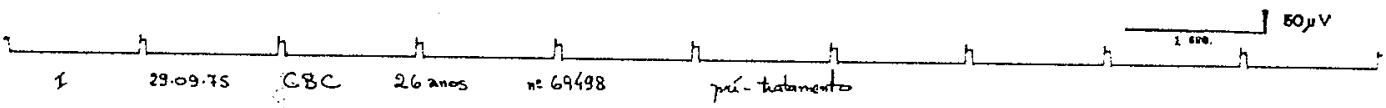

Fig. 5(a) - EEG sem anormalidades antes do tratamento

P.

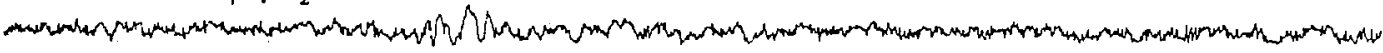

$F_{8} \cdot \varepsilon_{x}$

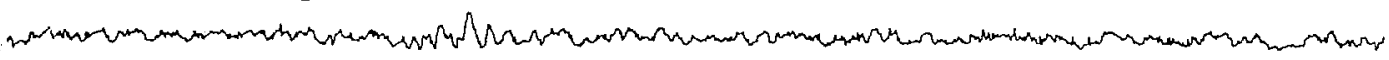

$T_{3} \cdot c_{2}$

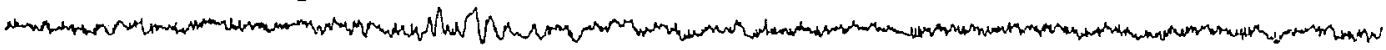

$T_{4}-c_{z}$

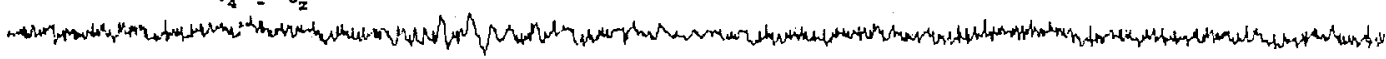

$0_{1} \cdot c_{2}$

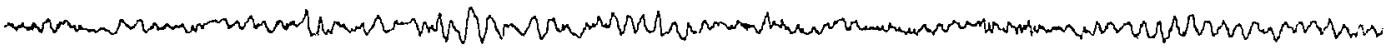

$0_{2}-c_{z}$

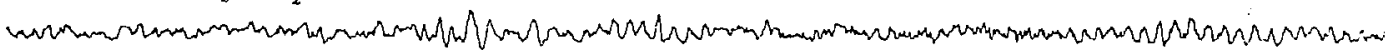

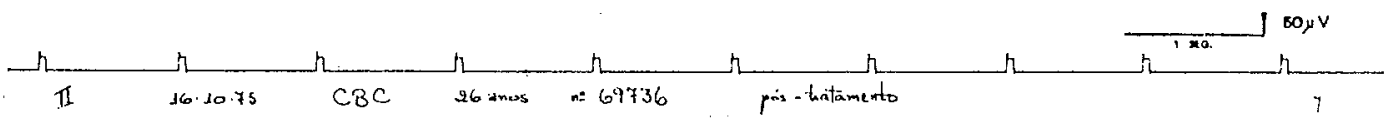

Fig. 5(b) - EEG mostrando sinais raros de anormalidades paroxística difusa frustra, mais ou menos 2 horas apos a medicação

CASO 3 - M.A.N.P., 38 anos, preta, feminina, portadora de forma intestinal. Não apresentava patologia intercorrente. Foi medicada, em jejum, com $750 \mathrm{mg}$ de oxamniquine. Três ho- ras após a medicação passou a apresentar vá rios episódios de vômitos. Medicada com antiemético parenteral, continuou apresentando vômitos que cessaram 4 horas após a tomada do 
CARVALHO, S. A. de; SHIKANAI-YASUdA, M. A.; AMATO NETO, V; SHIROMA, M. \& LUCCAS, F. J. C. - Neurotoxicidade do oxamniquine no tratamento da infecção humana pelo $\mathbf{S}$. mansoni. Rev. Inst. Med. trop. São Paulo 2\%: $132 \cdot 142,1985$.

oxamniquine. A paciente recebeu alimentação 5 horas após o tratamento, tendo recebido alta em bom estado geral. Ao deixar o Hospital a paciente apresentou tonturas e, imediatamente, seguida de desmaio com perda de consciência, sem abalos musculares. Foi reencaminhada à nossa clínica onde foi internada para observação. Não se precisou a duração da perda de consciência. Já na enfermaria a paciente apresentou novos episódios de vômitos e diarréia, tendo sido medicada sintomaticamente, tendo alta 24 horas depois, em bom estado geral e não apresentando nenhuma queixa. Por condiçöes operacionais, não foi feito o estudo eletro. encefalográfico.

CASO 4 - P.S., 29 anos, masculino, funcionário do Hospital das Clínicas, no setor de caldeiras. Portador de forma intestinal sem patologia intercorrente, ou queixas psiquiátricas pregressas. viedicado com $750 \mathrm{mg}$ de oxamniquine, apresentou queixas de sonolência e tonturas durante a observação hospitalar. Após este período de 4 horas teve alta, não apresentando qualquer queixa ou sintoma. Duas horas depois da alta, ou seja, 6 horas após a medicação, passou a apresentar quadro de confusão mental, amnésia, desorientação no tempo e espaço e verbalização desconexa. Embora o quadro decrecesse progressivamente em intensidade, as manifestações persistiram por mais ou menos 24 horas.

CASO 5 - M.A.N., 29 anos, branca, feminina, portadora de forma hepatointestinal, sem patologia intercorrente. Medicada com $750 \mathrm{mg}$ de oxamniquine, apresentou tonturas e sonolência no período de observação hospitalar, tendo alta aparentemente bem. Logo após (a paciente não precisou o tempo) passou a apresentar quadro de confusão mental, amnésia, tonturas e incoordenação motora à deambulação com defervescência progressiva e duração de mais ou menos 12 horas.

CASO 6 - R. S., 38 anos, preta, feminina, portadora de forma intestinal, sem patologia intercorrente ou queixas neuropsiquiátricas. Medicada com $875 \mathrm{mg}$ de oxamniquine, apresentou sonolência, tonturas, amnésia transitória e desorientação no tempo e espaço. A duração das manifestações foi de 6 a 8 horas.

CASO 7 - M.M.N., 30 anos, branca, feminina, portadora de forma hepatoesplênica compensa- aa, sem patologia intercorrente. Medicada com $500 \mathrm{mg}$ de oxamniquine, não apresentou efeitos colaterais durante a observação hospitalar. Por sugestão de terceiros, a paciente continuou a terapêutica por mais 5 dias ( $500 \mathrm{mg} /$ dia). Na sexta dose a paciente retornou ao ambulatório em mau estado geral, referindo que após a quarta dose $(2000 \mathrm{mg}$ ) passou a apresentar confusão mental, amnésia, tonturas, sensação de flutuação, incoordenação motora, náuseas, cólicas abdominais e diurese excessiva. Ao exame clínico, no dia seguinte à última dose (3000 mg) a paciente apresentou-se trêmula, com bradipsiquismo, amnésia e incoordenação motora à deambulação.

Dos 20 pacientes submetidos ao estudo eletroencefalográfico, $3(15 \%)$ apresentaram alterações no traçado (Fig. $3(a, b), 4(a, b), 5(a, b)$ ), sem contudo apresentarem manifestações de comprometimento neuropsiquiátricos.

\section{DISGUSSĀO}

A frequiência de indivíduos que apresentaram reações colaterais neuropsiquiátricas foi elevada. Essas reações foram, na maioria, leves e se caracterizaram principalmente por sonolência, tontura e cefaléia. Entretanto alguns individuos desenvolveram reações mais intensas, traduzidas principalmente por amnésia transitória, psicose tóxica e convulsão (Tabela V). Os dados de literatura são concordantes com os nossos, no que diz respeito aos efeitos colaterais de pequena intensidade, porém, pou$\cos$ são os relatos de neurotoxicidade grave com o emprego do oxamniquine, e, quando existentes, são imprecisos ou mal documentados. Alguns procuram até eximir o medicamento de tal toxicidade.

COUTINHO \& col. ${ }^{5}$, DOMINGUES \& COU. TINHO 6 , MOURA \& col. ${ }^{13}$ detectaram distúrbios neuropsiquiátricos (excitação psicomotora, alucinação, alterações đo comportamento) e alterações eletroencefalográficas, em indivíduos tratados com oxamniquine. Os Autores justificaram que tais manifestações seriam decorrentes de "estímulos inespecíficos" da droga em indivíduos com patologias neuropsiquiátricas preexistentes.

Outros Autores, realizando estudo eletroen. cefalográfico pré e pós-tratamento ao oxam- 
CARVALHO, S. A. de; SHIKANAI-YASUDA, M. A.; AMATO NETO, V.; SHIROMA, M. \& IUCCAS, F. J. C. - Neurotoxicidade do oxamniquine no tratamento da infecção humana pelo S. mansoni. Rev. Inst. Med. trop. São Paulo 27. 132-142, 1985.

niquine, não encontraram alterações que sugerissem uma possivel neurotoxicidade do medicamento (FOSTER ${ }^{7}$, SILVA \& col. ${ }^{16}$, DOMINGUES \& COUTINHO ${ }^{6}$, KATZ \& col. ${ }^{8,10}$, PRATA \& col. ${ }^{14}$. Entretanto, os primeiros indícios de uma possivel neurotoxicidade mais grave com o uso do oxamniquine começaram a surgir a partir de 1976.

CAMPOS \& col. ${ }^{2}$, SHIKANAI \& col. ${ }^{15}$, BINA \& SPINOLA ${ }^{1}$, KATZ \& col. ${ }^{4}$, CARVALHO ${ }^{3}$, KEYSTONE 12 e CARVALHO \& col. 4 , descreveram reações severas com o uso do oxamniquine no tratamento da esquistossomose, caracterizadas por: amnésia transitória, distúrbios de comportamento, excitação psicomotora, alteração da percepção visual, psicose tóxica e convulsão. Estes achados estão em concordância com os resultados obtidos neste trabalho confirmando a ação neurotóxica do oxamniquine. Por outro lado, torna-se necessário chamar a atenção para o emprego indiscriminado do medicamento, sem uma observação médica, pelo menos, nas quatro primeiras horas que se seguem à administração do medicamento, face à elevada incidência de efeitos neurotóxicos do oxamniquine.

\section{SUMMARY}

\section{Neurotoxicity of oxamniquine in the treatment of human infection due to $S$. mansoni}

One hundred and eighty patients from the "Clínica de Doenças Infecciosas e Parasitárias do Hospital das Clínicas da Faculdade de $\mathrm{Me}$ dicina da Universidade de São Paulo" with mansonic schistosomiasis have been treated with oxamniquine (single oral dose $12.5-15 \mathrm{mg}$ or $16-20 \mathrm{mg} / \mathrm{kg}$ body weight, respectively to patients younger or older than 15 years old). The patients were 5 to 65 years old and the predominant clinical forms were intestinal and hepato-intestinal disease. The main neuropsychiatric side effects were: drowsiness $(50.6 \%)$, dizziness $(41.1 \%)$, headache $(16.1 \%)$, temporary amnesia $(2.2 \%)$, behaviour disturbances $(1.7 \%)$, chills $(1.1 \%)$, seizures $(1.1 \%)$. In 20 patients the neurotoxicity associated with the drug has been evaluated comparing the electroencephalogram before and after the treatment. Alterations have been detected in $3(15 \%)$ but were not associated 'with neuropsychiatric manifes- tations. The results show that oxamniquine determines toxic side effects in the neuropsychiatric area.

\section{AGRADECIMENTOS}

Ao Dr. Aluísio Augusto Cotrim Segurado, Dr. Celso Francisco Hernandes Granato e Rudney Checco pela ajuda na realização do presen. te trabalho.

\section{REFERENCIAS BIBLIOGRAFICAS}

1. BINA, J. C. \& SPINOLA, A. - ConvuIsão associada ao uso do oxamniquine - relato de um caso. Rev. Soc. bras. IMed. trop. 10: 221-223, 1976.

2. CAMPOS, R.; CIMERMAN, B.; SILVA, N. P.; SALO MON, N. L. \& SAPIENZA, P. - Tratamento da esquistossomose mansônica pelo oxamniquine em região não endêmica. XXI Congresso da Sociedade Brasileira de Medicina Tropical e I Congresso da Sociedade Brasileira de Parasitologia. (Resumos), Belém, PA, 1976.

3. CARVALHO, S. A. - Aspectos epidemiológicos e quimioterápicos na esquistossomose mansônica. Contribuição ao estudo da quimioterapia pelo oxammiquine. São Paulo, Faculdade de Medicina da Universidade de São Paulo [Dissertação de Mestrado], 1978.

4. CARVALHO, S. A.; LUCCAS, F. J. C.; DELATORRE, M. C.; BATISTA. M. C. P.; MEIRA, J. A. \& AMATO NETO, V. - Estudo sobre a neurotoxicidade do oxamniquine. XV Congresso da Sociedade Brasileira de Medicina Tropical (Resumos), Campinas, SP, 1979.

5. COUTINHO, A.; DOMINGUES, A. L. C. \& BONFIM, J. R. A. - Tratamento da esquistossomose mansônica com oxamniquine. Rev. Inst. Med. trop. São Paulo 15 (Supl. 1): 104-119, 1973.

6. DOMINGUES, A. L. C. \& COUTINHO, A. - Tratamento da esquistossomose mansônica com oxamniquine oral. Rev. Inst, Med. trop. São Paulo 17: 164-180, 1975.

7. FOSTER, R. - The preclinical development of oxamniquine. Rev. Inst. Med. trop. São Paulo 15 (Supl. 1) I-9, 1973

8. KATZ, N.; GRIMBAUM, E.; CHAVES, A.; ZICHER F. \& PELLEGRINO, J. - Clinical trials with oxamniquine by oral route in schistosomiasis. Rev. Inst. Med. trop. São Paulo 18: 317-377, 1976.

9. KATZ, N.; ROCHA, R. S.; OLIVEIRA, V. B. \& PE REIRA, J. P. - Ensaios clínicos com oxamniquine em áreas endêmicas de esquistossomose mansoni. XIII Congresso da Sociedade Brasileira de Medicina Tropical e II Congresso da Sociedade Erasileira de Parasitologia (Resumos). Brasília, DF, 1977.

10. KATZ, N.; ZICHER, F. \& PEREIRA, J. P. - Field trials with oxamniquine in a schistosomiasis mansoni endemic area. Am. J. trop. Med. Hyg. 26: 234-237, 1977. 
CARVAIFH, S. A. de; SHIKANAI-YASUdA, M. A.; AMATO NETO, V.; SHIROMA, M. \& LUCCAS, F. J. C. - NeurotoxicIdade do oxamniquine no tratamento da infeç̧ão humana pelo $S$. mansoni. Rev. Inst. Med. trop. São Paulo 27: $132 \cdot 142,1985$.

11. KAYE, B. \& WOOLHOUSE, N. M. - The metabolism of oxamniquine a new schistosomicide. Am. trop. Med. Parasit. 70: 323-328, 1976.

12. KEYSTONE, J. S. - Seizures and electroencephalograph changes associated with oxamniquine therapy. Amer. J. trop. Med. Hyg. 27: 360-362, 1978.

13. MOURA, H.; VISQUIER, H.; BECHARA, S.; DOMINGUES, A. L. C. \& COUTINHO, A. - Tratamento da esquistossomose mansônica em crianças com oxamniquine sob a forma de xarope. Rev. Soc. bras. Med. trop. 10: 249-259, 1976.

14. PRATA, A.; LAURIA, L.; FIGUEIREDO, J. F. M. \& SENNA, P. G. - Tratamento da esquistossomose mansoni pelo oxamniquine em dose única, pela via oral. Rev, Soc. bras. Med. trop. 10: 127-136, 1976.
15. SHIKANAI-YASUDA, M. A.; CARVALHO, S. A.; LUCCAS, F. J. C.; SHIROMA, M. \& FERREIRA, J. M. Ingestão excessiva de oxamniquine. XII Congresso da Sociedade Brasileira de Medicina Tropical e I Congresso da Sociedade Brasileira de Parasitologia (Resumos). Belém, PA, 1976.

16. SILVA, L. C.; SETTE JR., H.; CHAMONE, D. A. F.; ALQUEZAR, A. S. \& MONTEIRO, A. A. - Oxamniquine (UK-4271) no tratamento da esquistossomose mansônica em área não endêmica. Rev̀. Inst. Med. trop. São Paulo 15: 143-147, 1973.

Recebido para publicação em $7 / 8 / 1984$. 